\title{
Seguridad Alimentaria y Patrimonio Gastronómico: Una Aproximación Politécnica a la Vinculación con la Sociedad.
}

\author{
Teresa Erice, $\mathrm{MPhil}^{1}$, Cecilia Banderas Avilés, $\mathrm{MSc}^{2}$ \\ ${ }^{1}$ Universidad Del Pacífico, Ecuador, teresa.erice@upacifico.edu.ec \\ ${ }^{2}$ Escuela Superior Politécnica del Litoral, Ecuador, jcbander@espol.edu.ec
}

\begin{abstract}
Resumen- El proyecto de vínculos con la sociedad "Recuperación y Difusión del Patrimonio Gastronómico de la Ruta del Cacao en los Cantones de Naranjal y Balao"(Guayas, Ecuador) explora el potencial de aplicar metodologías de investigación antropológica para la educación fuera del aula. En este caso se realizó el proyecto con alumnos de Turismo de la Escuela Superior Politécnica del Litoral (ESPOL, Guayaquil, Ecuador). Presentamos esta metodología como marco para involucrar a alumnos de escuelas politécnicas en temas sociales a través de la antropología, un campo de conocimiento generalmente ausente del currículum politécnico.
\end{abstract}

Palabras-clave- Vinculación, Sociedad, Educación, Antropología, Gastronomía.

Abstract-Educational Outreach in Ecuadorian Universities is carried out mainly through the so-called 'Proyectos de Vínculación con la Sociedad'. The project presented here recorded and disseminated gastronomic heritage of two small localities (Naranjal and Balao) in the Guayas province, Ecuador, by training students from the Escuela Superior Politécnica del Litoral (ESPOL) in anthropological methods of research. This approach is presented as a framework for involving students in social issues, but also as a means to introduce non-traditional fields of knowledge in the polytechnic curriculum.

Keywords- Educational Outreach, Society, Anthropology, Gastronomy.

\section{INTRODUCCIÓN}

Recientemente en 2015 se establecieron los 17 Objetivos de Desarrollo Sostenible (ODS) para la Agenda 2030 de la ONU. La sostenibilidad no es alcanzable sin la innovación y la educación. Siguiendo esta línea, presentamos una metodología innovadora a aplicar en proyectos de vinculación con la sociedad en escuelas politécnicas, que implica fusionar conocimiento científico y social a través del trabajo de campo antropológico. En este caso, se utilizó la gastronomía como elemento de unión entre nutrición, higiene y patrimonio cultural.

Los vínculos con la sociedad se han aproximado tradicionalmente desde la educación politécnica como una transferencia de tecnologías y capacitación de la universidad a las comunidades más necesitadas. Sin embargo, la palabra vínculo implica una relación y por tanto, debería existir transferencia en ambas direcciones. El proyecto Recuperación y Difusión del Patrimonio Gastronómico de la Ruta del Cacao

Digital Object Identifier (DOI): http://dx.doi.org/10.18687/LACCEI2016.1.1.327 ISBN: 978-0-9822896-9-3

ISSN: 2414-6390 en los Cantones de Naranjal y Balao, dirigido por C. Banderas, es innovador en este sentido. Por un lado sigue una línea de capacitación nutricional y difusión de buenas prácticas alimentarias, desarrollado en proyectos anteriores de la Facultad de Ingeniería Marítima, Ciencias Biológicas, Oceánica y Recursos Naturales de la ESPOL, Ecuador. Se firmó convenios con los gobiernos municipales de Naranjal y Balao (Prov. Guayas, Ecuador) que incluían capacitaciones en buenas prácticas en la manipulación de alimentos y ferias gastronómicas, que difundieran el rol fundamental de la gastronomía tradicional en la educación alimentaria. Pasar de un enfoque puramente nutricional a uno gastronómico, abrió las puertas del proyecto a campos de conocimiento, que normalmente no se asocian a la educación politécnica, como por ejemplo, el patrimonio cultural. El proyecto capacitó a los alumnos involucrados en el proyecto en técnicas de investigación antropológica, para que ellos mismos fueran los protagonistas de un inventario de prácticas gastronómicas en los dos cantones. Estos inventarios consistían en la recopilación de tradiciones orales gastronómicas, es decir, platos y recetas populares poco conocidas fuera de las localidades. El proyecto presenta el patrimonio gastronómico como un vínculo común entre comunidades rurales y urbanas y entre clases sociales distintas de la provincia del Guayas. Es por ello que la labor de difusión del patrimonio gastronómico de Naranjal y Balao transcendió los límites de los cantones, contando con una importante colaboración interinstitucional, fundamental en la consecución de proyectos culturales con fondos limitados, en particular la de la Dirección de Turismo de la Prefectura del Guayas y del Archivo Histórico del Guayas. En resumen, este proyecto transfirió conocimiento de la universidad a la comunidad en seguridad alimentaria, recuperó conocimiento cultural de interés patrimonial para el conjunto de la región y llevó a la educación politécnica más allá de la tecnología, para lograr una mayor involucración en temas sociales.

\section{REVISIÓN DE LA LITERATURA}

En la actualidad, la seguridad alimentaria no sólo se limita a terminar con el hambre (segundo Objetivo de Desarrollo Sostenible de la Agenda 2030), sino que incluye otros elementos relacionados con la gastronomía, como el comercio, el procesamiento, el comportamiento humano y el acceso y consumo de alimentos diversos [1]. La gastronomía

$14^{\text {th }}$ LACCEI International Multi-Conference for Engineering, Education, and Technology: "Engineering Innovations for Global Sustainability”, 20-22 July 2016, San José, Costa Rica. 
sostenible, en cambio, apunta a disminuir las diferencias sociales en la mesa y a incrementar la apreciación hacia la calidad de la comida como un elemento importante de bienestar individual, biológico y social [2]. Ambos conceptos se pueden relacionar de diferentes maneras: en la producción local de alimentos, la gastronomía casera, la transmisión de tradiciones culinarias entre generaciones, el derecho a la diversidad de alimentos y el impacto del turismo gastronómico sobre el desarrollo local [2].

Todas estas cuestiones se abordan desde el proyecto de vinculación con la sociedad Recuperación y Difusión del Patrimonio Gastronómico de la Ruta del Cacao en los Cantones de Naranjal y Balao. Dentro de la clasificación que ofrece L. Malagón Plata sobre los diferentes tipos de vinculación entre la universidad y la sociedad, se podría incluir este proyecto dentro de los "procesos de construcción curricular a través de la vinculación con el entorno [3]”. Sin embargo, ¿qué tipo de construcción curricular? Hace unos años H. Gallegos ya alertó que la mayoría de programas universitarios en Ingeniería en Latinoamérica no eran conscientes de que sus currículos se enfocaban demasiado, o casi exclusivamente, en procesos y habilidades técnicas [4]. El s. XXI requiere de ingenieros que sean capaces de liderar e innovar en contextos sociales complejos, interdisciplinares y multi-culturales; deben entender y participar de la cultura que les rodea, a nivel empresarial y productivo, pero también social [5], [6]. Es lo que se promueve desde el Holistic Engineering. Interesar a alumnos universitarios en temas, diferentes a los que han escogido, no es tarea fácil, por lo que introducir la interdisciplinariedad desde los primeros semestres es importante [6]. No sólo es importante, sino necesario, para que los alumnos puedan en niveles posteriores llevar esta interdisciplinariedad a la práctica, por ejemplo, a través de proyectos de vinculación con la sociedad.

\section{LA VINCULACIÓN CON LA SOCIEDAD}

\section{A. Antecedentes}

Como antecedente directo de este proyecto cabe mencionar el proyecto de vínculos Mi Loncherita Saludable, dirigido por C. Banderas en 2014 [7]. En este proyecto se unieron dos carreras de la Facultad de Ingeniería Marítima, Ciencias Biológicas, Oceánica y Recursos Naturales de la ESPOL para la educación en seguridad alimentaria de un colegio. Después de un análisis y medición de las condiciones nutricionales en el Colegio Luis Enrique Morales Alfaro de la parroquia de Chongón en Guayaquil (Ecuador), se identificaron problemas en prácticas higiénicas y educación nutricional. Para remediar el problema, se llevaron a cabo capacitaciones y charlas de sensibilización. Los alumnos universitarios de la ESPOL que participaron en este proyecto, abordaron temas relacionados con la bioseguridad alimentaria; el balance nutricional de los alimentos; buenas prácticas de manipulación de alimentos; la composición de grasas, carbohidratos y vitaminas de los alimentos; y recetas apropiadas para una lonchera escolar. El escenario escolar se presentó como apropiado para el diseño de iniciativas que inserten a los alumnos universitarios en el contacto con situaciones y problemas reales [7] a través de la metodología APS (Aprendizaje Servicio) [8].

Partiendo de esta experiencia, se reflexionó sobre la naturaleza del concepto de "vínculo con la sociedad". La implicación del universitario, en este caso, estudiante de la Escuela Superior Politécnica del Litoral, en los problemas reales del entorno social es sólo el primer paso. El alumno debe ser también consciente de aquello que le pueda aportar la sociedad y que contribuye a su aprendizaje continuo. El aprendizaje de por vida es la mejor vía por la que educar en la sostenibilidad y responsabilidad social. Sin alejarnos de las experiencias positivas recabadas en vinculación social en colegios, decidimos llevar a los estudiantes universitarios a otros escenarios, de educación informal, en donde no existiera una clara relación entre la persona que imparte el conocimiento y la persona que lo recibe. La vinculación, por tanto, la llevamos al terreno de la relación y el intercambio recíproco de conocimiento. Consideramos que el método de investigación antropológico sería el ideal para involucrar de manera respetuosa al alumno en costumbres y cultura local de zonas rurales de la provincia del Guayas. Es aquí, donde la capacitación en seguridad alimentaria se integra con la valorización y comunicación del patrimonio gastronómico.

\section{B. Patrimonio gastronómico, educación y políticas nacionales/ internacionales}

La vinculación con la sociedad, conjuntamente con la docencia y la investigación, conforman los tres pilares de la educación universitaria ecuatoriana. Según el principio de pertinencia, establecido en el Art. 107 de la LOES (Ley Orgánica de Educación Superior), la educación debe "responder a las expectativas y necesidades de la sociedad, a la planificación nacional, y al régimen de desarrollo, a la prospectiva de desarrollo científico, humanístico y tecnológico mundial y a la diversidad cultural [9]". Sin embargo, ¿cómo juntar todos estos elementos?

La Antropología, como disciplina, está creciendo como contenido transversal en el currículo universitario, incluso crecen el número de carreras que lo integran dentro de la malla curricular como asignatura básica. Como ejemplo, podemos citar las nuevas carreras de Ingeniería en Sistemas y en Recursos Renovables de la Universidad Del Pacífico-Ecuador, que forman a sus alumnos, no sólo en conocimientos generales de gerencia y emprendimiento, sino también en desarrollo sostenible y antropología, como ejes fundamentales en la formación de ingenieros capaces de innovar económicamente y tecnológicamente de manera comprometida con la sociedad.

Sin embargo, no es estrictamente necesario crear materias dentro de una malla curricular para introducir contenidos interdisciplinares. Los proyectos de vínculos con la sociedad sirven este propósito, para que el alumno de una carrera de ingeniería, o cualquier otra, integre conocimientos propios de su carrera con otras disciplinas y los aplique a problemas

$14^{\text {th }}$ LACCEI International Multi-Conference for Engineering, Education, and Technology: "Engineering Innovations for Global Sustainability", 20-22 July 2016, San José, Costa Rica. 
sociales [10]. Es esto, en fin, lo que se promueve desde la Agenda 2030 para la Sostenibilidad, cuando se recalca que los distintos ODS deben abordarse de manera integrada. No se puede considerar la producción y consumo responsable, sin la educación, la innovación y la erradicación de la pobreza. Sin embargo, es éste último objetivo el que mayor desafíos representa, sobre todo para los países en vías de desarrollo [11].

Empoderar a la población de su riqueza patrimonial tiene una incidencia directa sobre la transformación de la matriz productiva del país, muchas veces de maneras inesperadas, puesto que elementos culturales, también pueden convertirse, de manera innovadora, en productos y servicios.

La Convención sobre la Protección y Promoción de la Diversidad de las Expresiones Culturales tiene como uno de sus objetivos principales "reafirmar la importancia del vínculo existente entre la cultura y el desarrollo para todos los países en desarrollo, y apoyar las actividades realizadas en el plano nacional e internacional para que se reconozca el auténtico valor de ese vínculo [12, Art. 1, f]". La misma convención define las actividades, bienes y servicios culturales como "las actividades, bienes y servicios que, considerados desde el punto de vista de su calidad, utilización o finalidad específicas, encarnan o transmiten expresiones culturales, independientemente del valor comercial que puedan tener. Las actividades culturales pueden constituir una finalidad de por sí, o contribuir a la producción de bienes o servicios culturales [12, Art 4.4]".

Con respecto a las políticas nacionales, dentro del Plan Nacional de Buen Vivir (PNBV), el objetivo 5 establece la necesidad de "construir espacios de encuentro común y fortalecer la identidad nacional, las identidades diversas, la plurinacionalidad y la interculturalidad [13]". Asimismo, el objetivo 10 especifica la necesidad de impulsar la transformación de la matriz productiva [13]. La valoración del patrimonio tangible e intangible puede vincularse a la dinamización de la economía por medio de la actividad turística. De hecho, así lo entiende también la UNESCO, a través de su Plan de Acción de Patrimonio Mundial y Turismo Sostenible [14]. Sin embargo, el turismo cultural no se puede promocionar sin primero potenciar su valor dentro de la misma población que lo vive y lo produce. Estas consideraciones se encuentran actualmente en debate para el Plan de Acción para el Patrimonio Mundial en América del Sur 2015-2020 [15]. Es por tanto de vital importancia, como lo especifica el objetivo 5 del PNBV, encontrar elementos comunes entre las identidades diversas ecuatorianas para fortalecer la identidad nacional. La gastronomía se presenta en este proyecto como un elemento cohesionador y de gran accesibilidad para potenciar el patrimonio cultural inmaterial y popular [16].

\section{Colaboración inter-institucional.}

El último ODS planteado en la Agenda 2030 de la ONU es el de crear "Alianzas para lograr los objetivos" [11]. La consecución de este objetivo es la única manera viable de integrar distintas disciplinas, instituciones y campos de acción con un mismo fin. La vinculación con la sociedad, en principio, sólo requiere de la colaboración de una universidad con una comunidad local, a través de un convenio. Sin embargo, las posibilidades de colaboración inter-institucional son infinitas. Para el proyecto Recuperación y Difusión del Patrimonio Gastronómico de la Ruta del Cacao en los Cantones de Naranjal y Balao se contó con la colaboración de los gobiernos municipales de Naranjal y Balao, pero también con la implicación desinteresada y comprometida de la Dirección de Turismo de la Prefectura del Guayas y del Archivo Histórico del Guayas. La dificultad de encontrar financiación para fines sociales no es una novedad para muchas universidades o instituciones públicas y privadas. A través de la colaboración inter-institucional, no sólo se consiguen abordar los Objetivos del Desarrollo Sostenible de manera integrada, sino que se reparte la responsabilidad y carga financiera. Cabe recalcar que éste no es recurso de colaboración privativo de la vinculación de la sociedad o novedoso de la Agenda 2030. La colaboración interinstitucional es la razón fundamental por la que en Ecuador han prosperado y crecido en los últimos años proyectos de toda índole con fines interdisciplinares [17].

\section{METODOLOGÍA E IMPLEMENTACIÓN}

Según se desprende del título, el proyecto consta de dos partes: la recuperación y la difusión. Por tanto, la metodología es diferente, aunque complementaria para estas dos partes. Toda fase del proyecto, estuvo a cargo de equipos de trabajo y nunca de una sola persona (ver Tabla I). Cada grupo estuvo compuesto por tres estudiantes.

Para la recuperación del patrimonio gastronómico de los cantones de Balao y Naranjal en la Ruta del Cacao, se implementó el método antropológico de investigación. "Un rasgo clave de la antropología es que tiene una dimensión transcultural y comparativa [18]". El ser humano construye su identidad a partir de diferenciación o de la comparación con 'el otro' [19]. Esta toma de conciencia de lo 'diferente' puede producirse tanto en el espacio (etnografía), como en el tiempo (etnología) [20]. Un estudio antropológico coherente, debe abarcar ambas dimensiones. Para ello, en la fase de recuperación, se crearon grupos de trabajo, de tres estudiantes cada uno (ver Tabla I), que cubrieran, tanto el pasado (investigación bibliográfica) como el presente (observación de campo y entrevistas cualitativas) [20]. Para una aproximación correcta a la observación de campo y la realización de entrevistas, los alumnos recibieron formación previa en las aulas.

La investigación bibliográfica tuvo como base los repositorios bibliográficos de Guayaquil, empezando por el Archivo Histórico del Guayas, que guarda abundante documentación histórica sobre la producción del cacao y fotos antiguas de la provincia (ver Tabla I). 
Para la observación de campo, en cambio, los alumnos contaron con la guía de representantes de la Dirección de Turismo de la Prefectura del Guayas en el recorrido de ubicaciones claves a lo largo de la Ruta del Cacao en Naranjal y Balao, con el fin de documentar en diarios de campo la cultura gastronómica latente en la actualidad (ver Fig. 1). Los diarios de campo se complementaron con material de documentación digital (ver Tabla I).

Las entrevistas cualitativas se enfocaron en recuperar recetas de transmisión oral, así como testimonios de la población local, sobre todo la de más avanzada edad (ver Tabla I). Estas entrevistas se realizaron en la modalidad informal, de manera espontánea [20]. Sin embargo, no por ello se dejaron de planificar con guiones, documentar mediante grabaciones y transcribir para su conservación y su posterior difusión. El entrenamiento en la realización de entrevistas cualitativas no sólo conciencia al estudiante en la conservación del patrimonio, sino también trabaja sus habilidades en la comunicación asertiva [20].

Para la difusión de los resultados de investigación se unió el componente antropológico con las técnicas culinarias en varios eventos: ferias gastronómicas, charlas en 'Buenas prácticas en la manipulación de Alimentos y Bebidas', la realización de un simposio y la elaboración de una publicación de difusión (ver Tabla I). Una vez más, los estudiantes se dividieron en grupos de tres. La difusión se realizó en tres niveles (local, provincial y nacional). La difusión local fue de carácter específica, dirigida a actores clave en la conservación y difusión del patrimonio gastronómico en Naranjal y Balao y la educación en buenas prácticas alimentarias; la provincial fue de carácter general (público variado) a través de la realización de un simposio auspiciado por el Archivo Histórico del Guayas; y la difusión nacional consistió en la publicación de un dossier digital titulado Guayas: Entre sabores y saberes Patrimoniales. Naranjal y Balao (ver Fig. 2) $[21]$

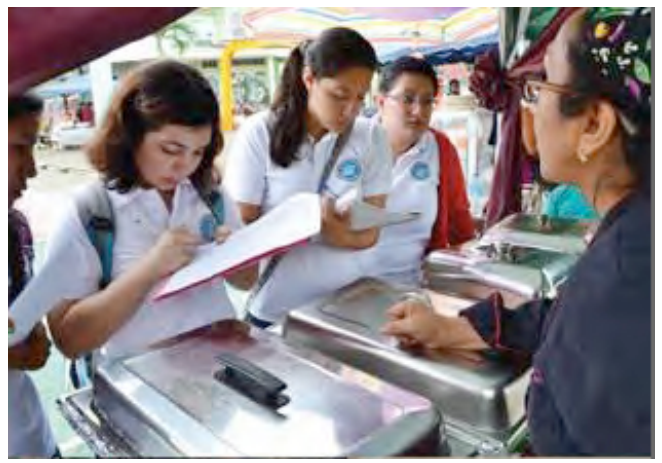

Fig. 1 Alumnas de la ESPOL. De izquierda a derecha: N. Coello, M. Andrade, L. Loor. Feria Gastronómica de Naranjal 2015. Fotografía por M. Icaza (ESPOL) [21].
La difusión dirigida (o local) en primer lugar consistió en la preparación e impartición de charlas sobre buenas prácticas por parte de los estudiantes de la ESPOL a los prestadores de servicios gastronómicos de los dos cantones (Naranjal y Balao) (ver Tabla I). La clausura a nivel local se llevó a cabo en espacios cedidos por los municipios, donde se celebró un festival gastronómico (uno en cada cantón), de la mano de los estudiantes y de los mismos prestadores de servicios gastronómicos capacitados.

La difusión general (o provincial) constó de la planificación y ejecución de un simposio acorde al propósito del proyecto en las instalaciones del Archivo Histórico del Guayas, Guayaquil, que llevó el nombre de Primer Simposio Gastronómico del Guayas. Memoria e Identidad Cultural, celebrado el 27 de enero de 2016 (ver Tabla I). En este segundo nivel de difusión se fortalecieron los vínculos entre la ciudad y su periferia rural, llevando la riqueza gastronómica y patrimonial de dos localidades poco conocidas al centro de la ciudad.

La difusión continua (o nacional) es la que se encargó de difundir los objetivos del proyecto más allá de su fecha de cierre. Se compilaron los informes de actividades de los cantones y se elaboró la antes mencionada publicación de difusión, elaborada por los alumnos en formato de dossier digital (ver Tabla I y Fig. 2). El contenido del mismo es un fiel reflejo de la naturaleza interdisciplinar del proyecto, pues combina la recuperación de recetas locales del ámbito rural de la provincia del Guayas con recomendaciones para la buena práctica alimentaria.

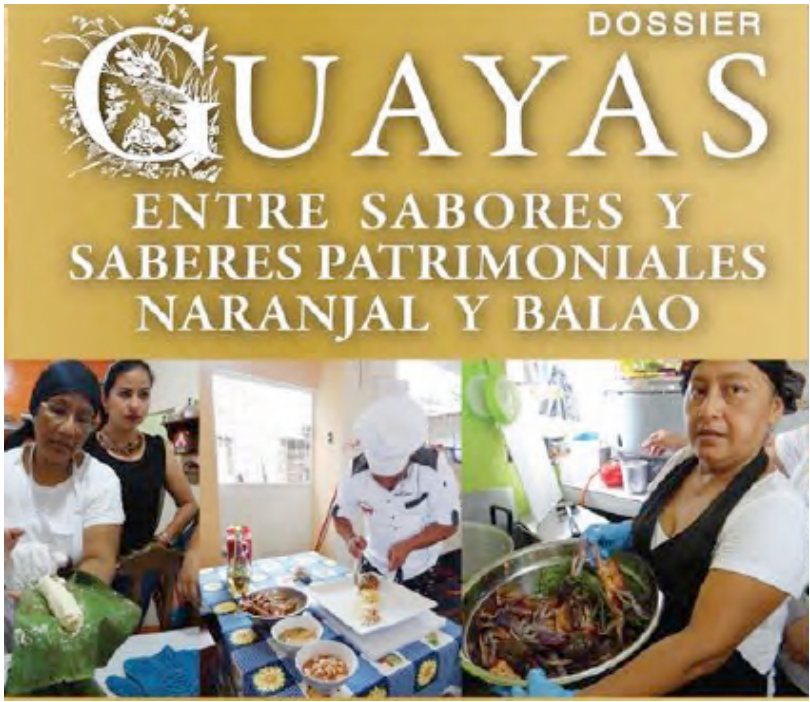

Fig. 2 Dossier digital, Guayas: Entre sabores y saberes Patrimoniales. Naranjal y Balao. Parte de la portada de la publicación, diseño de M. Bravo (Archivo Histórico del Guayas) y fotografías por M. Icaza (ESPOL) [21]. 
TABLA I

ACTIVIDADES Y RESULTADOS POR GRUPOS DE ESTUDIANTES

\begin{tabular}{|c|c|c|}
\hline \multirow[t]{2}{*}{ Grupos } & \multicolumn{2}{|c|}{ Recuperación } \\
\hline & Actividades & Resultados \\
\hline 1 & $\begin{array}{l}\text { Investigación en fondos } \\
\text { bibliográficos. } \\
\text { Investigación en fondos } \\
\text { fotográficos. } \\
\text { Compilación de la } \\
\text { información. }\end{array}$ & $\begin{array}{l}\text { Fichas bibliográficas de recetas } \\
\text { antiguas. } \\
\text { Catálogo de fotografías } \\
\text { antiguas. } \\
\text { Dossier: } \\
\text { - Introducción. } \\
\text { - Marco Histórico de } \\
\text { nuestra gastronomía } \\
\text { costeña. }\end{array}$ \\
\hline 2,3 & $\begin{array}{l}\text { Elaborar diarios de campo } \\
\text { que recojan la investigación } \\
\text { sobre la gastronomía actual, } \\
\text { y aquella que constituye } \\
\text { parte del pasado en los } \\
\text { cantones de Naranjal y } \\
\text { Balao. } \\
\text { Compilar información. }\end{array}$ & $\begin{array}{l}\text { Fichas técnicas de platos. } \\
\text { Inventario de la gastronomía } \\
\text { actual y pasada. }\end{array}$ \\
\hline 4,5 & $\begin{array}{l}\text { Investigación cualitativa } \\
\text { dirigida principalmente a la } \\
\text { población de mayor edad en } \\
\text { los cantones de Naranjal y } \\
\text { Balao, mediante entrevistas } \\
\text { informales. } \\
\text { Compilar información. }\end{array}$ & $\begin{array}{l}\text { Inventario de productos, } \\
\text { utensilios y cultura } \\
\text { gastronómica. }\end{array}$ \\
\hline & \multicolumn{2}{|c|}{ Difusión } \\
\hline & Actividades & Resultados \\
\hline 6,7 & $\begin{array}{l}\text { Preparar e impartir charlas - } \\
\text { taller sobre "Buenas } \\
\text { Prácticas en la Manipulación } \\
\text { de Alimentos y Bebidas" en } \\
\text { Naranjal y Balao a los } \\
\text { prestadores de servicios } \\
\text { gastronómicos. } \\
\text { Diseño de un pequeño } \\
\text { manual de buenas prácticas } \\
\text { en la manipulación de } \\
\text { alimentos. } \\
\text { Diseñar actividades lúdicas } \\
\text { para la retroalimentación. }\end{array}$ & $\begin{array}{l}\text { Prestadores de servicios } \\
\text { gastronómicos capacitados en } \\
\text { manipulación de alimentos y } \\
\text { bebidas. }\end{array}$ \\
\hline 8,9 & $\begin{array}{l}\text { Organización de ferias } \\
\text { gastronómicas. }\end{array}$ & Difusión local y provincial. \\
\hline $1,4,5$ & $\begin{array}{l}\text { Organización del Primer } \\
\text { Simposio Gastronómico del } \\
\text { Guayas "Memorias e } \\
\text { Identidad Cultural". }\end{array}$ & Difusión provincial y nacional. \\
\hline $\begin{array}{l}1,2,3 \\
4,5,6 \\
7,8,9\end{array}$ & $\begin{array}{l}\text { Diagramación, diseño y } \\
\text { redacción del dossier } \\
\text { "Guayas: Entre sabores y } \\
\text { saberes Patrimoniales. } \\
\text { Naranjal y Balao." }\end{array}$ & Difusión nacional. \\
\hline
\end{tabular}

\section{RESULTADOS}

Los estudiantes capacitaron a prestadores de servicios gastronómicos en dos municipalidades rurales. Entrevistaron y recuperaron memoria oral referente a gastronomía tradicional de catorce miembros de las comunidades de Naranjal y Balao (Guayas, Ecuador). Impartieron conocimiento referente a buenas prácticas y seguridad alimentaria, pero también difundieron conocimiento rural a nivel regional y nacional a través de eventos y una publicación de difusión [21], es decir, hubo un intercambio bidireccional de conocimiento. Los estudiantes salieron de su zona de confort académica y pusieron en práctica nuevas formas de interactuar con el entorno, en este caso, a través del método de investigación antropológico. Adicionalmente, para aquellos involucrados en la redacción, diseño y diagramación del dossier digital, fue una primera aproximación a la publicación basada en investigación.

Como resultados de aprendizaje podemos destacar la capacidad de trabajar como parte de un equipo multidisciplinar y la habilidad de asesorar comunidades y emprendedores en cuanto al desarrollo de una oferta de productos respetuosos con la naturaleza, del entorno social y cultural local. Éstos son resultados de aprendizaje comunes tanto para carreras politécnicas como de las ciencias sociales y humanidades. Son resultados de aprendizaje que deben adquirir los líderes y profesionales innovadores del s. XXI independientemente de la carrera que cursen.

\section{DISCUSIÓN}

El proyecto de vínculos con las sociedad Recuperación y Difusión del Patrimonio Gastronómico de la Ruta del Cacao en los Cantones de Naranjal y Balao ofrece como tesis la promoción del patrimonio gastronómico como herramienta para la valorización de la identidad local y la promoción de la cultura como producto y servicio. Adicionalmente, su metodología se propone como idónea para su implementación como elemento interdisciplinar y complementario en la educación universitaria politécnica fuera de las aulas.

El desconocimiento y la poca valoración del patrimonio gastronómico de los cantones de Balao y Naranjal requiere del esfuerzo colectivo de múltiples administraciones e instituciones. Este proyecto contribuyó a este fin, proponiendo la solución de este problema a través de la promoción y revalorización del patrimonio intangible de la provincia. Más específicamente, se otorgó visibilidad al patrimonio intangible gastronómico tradicional de Balao y Naranjal. Mediante la puesta en valor del patrimonio gastronómico de la Ruta del Cacao, se juntó a personas de distintos grupos sociales y geográficos en eventos culturales que promocionaron la gastronomía como un elemento cohesionador de identidad nacional y colectiva. Los asistentes aprendieron del concepto de patrimonio inmaterial, seguridad alimentaria y a valorar la cultura popular propia. Además, con la publicación del dossier digital sobre la gastronomía 
patrimonial de estos dos cantones, se pretende hacer conocer este potencial turístico dentro y fuera del país.

"Cultura popular" es un término que anteriormente se asociaba al concepto de folklor, pero que actualmente se acerca más al de 'patrimonio intangible' [22]. Según la Convención para la salvaguarda del patrimonio cultural inmaterial la gastronomía entraría dentro de dos categorías: conocimientos y usos relacionados con la naturaleza y el universo; y técnicas tradicionales y ancestrales [23]. La gastronomía es sin lugar a duda, uno de los productos sincréticos más visibles y accesibles para la comunidad, donde se funden saberes ancestrales con experiencias propias de la historia y sociedad ecuatoriana. Desde el 2010, el gobierno ecuatoriano ha reconocido la importancia de los saberes ancestrales aplicados a la ciencia y tecnología [24]. Sin embargo, es en el ámbito de la diversidad de expresiones culturales, donde inciden estos saberes con más fuerza. Mediante una recuperación y difusión del patrimonio gastronómico de Naranjal y Balao, se pueden crear las bases para fomentar vías innovadoras en la prestación de servicios al conjunto de la población residente y visitante de estos dos cantones.

\section{CONCLUSIÓN}

La sostenibilidad global es un fin, que sólo será alcanzable a través del fortalecimiento de la calidad de vida, salud, educación y erradicación de la pobreza. Es necesario educar a nuestros futuros profesionales en la integración de saberes y la capacidad de trabajar de manera integrada con otras disciplinas. Para ello es importante incorporar en el currículo mecanismos mediante los cuales estudiantes de ingenierías puedan, no sólo experimentar problemas sociales de primera mano, sino participar y formarse en entornos de educación no formal. La vinculación de la universidad con la sociedad debería ser el reflejo de una relación bilateral, no sólo unilateral. A través de la recuperación, mediante el trabajo de campo antropológico, del patrimonio gastronómico, entendido como un elemento de cultura popular compartido por diversos grupos sociales, se fomenta la valorización de la diversidad cultural, desde un punto de vista innovador, tanto para las comunidades como para los estudiantes de ingenierías.

\section{AGRADECIMIENTO}

Agradecemos en primer lugar la colaboración desinteresada del Archivo Histórico del Guayas y de la Dirección de Turismo de la Prefectura del Guayas, sin cuya valiosa ayuda no hubiera sido posible realizar el proyecto. Gracias al recibimiento por parte de la población y autoridades de los Gobiernos Autónomos Descentralizados de Naranjal y Balao y a la Unidad de Vinculación de la ESPOL por admitir este proyecto. Por último, este proyecto pertenece y fue realizado por los estudiantes de Alimentos y Bebidas I de la carrera de Turismo de la ESPOL, Ecuador, sin olvidar a los alumnos de la clase de Etnografía y Folklor de la misma carrera en el segundo periodo académico 2014-2015, en cuya experiencia, curiosidad y dedicación se inspiró este proyecto.

\section{REFERENCIAS}

[1] M. Wahlqvist, "Requirements for healthy nutrition: integrating food sustainability, food variety and health" J. Food Sci., vol. 69, no. 1, pp. 1618 , enero 2004.

[2] R. Scarpato, "Sustainable gastronomy as a tourist product," en Tourism and Gastronomy, A. Hjalajer y G. Richards, Eds. Londres: Routledge, 2002, pp. 132-152, p. 140.

[3] L. Malagón Plata, Universidad y Sociedad. Pertinencia y Educación Superior, Bogotá: Alma Mater Magisterio, 2004, p. 83.

[4] H. Gallegos, "The education of an engineer in a holistic age: a Latin American perspective," en Holistic Engineering Education. Beyond Technology, D. Grasso y M. Brown Burkins, Eds. Nueva York: Springer, 2010, pp. 99-112, p. 99.

[5] D. Grasso y M. Brown Burkins, Eds., Holistic Engineering Education. Beyond Technology, Nueva York: Springer, 2010.

[6] J. MacDougall, A. Ham y R. Pop-Iliev, "Enhancing the engineering curriculum through holistic engineering," en Examining Future Directions for Engineering Education: Proc. of the $3^{\text {rd }}$ Annual Canadian Engineering Education Association Conf., CEEA12, Winnipeg, MB, Canadá, junio 1720, 2012, W. Kinsner, Ed., Winnipeg: CEEA, 2012, pp. 312-317.

[7] C. Banderas Avilés y A. Quinteros Trelles, "El aprendizaje servicio en la evaluación de los resultados de aprendizaje. Un beneficio compartido con la comunidad," en Engineering Education Facing the Grand Challenges, What Are We Doing?: Proc. of the $13^{\text {th }}$ Latin American and Caribbean Conference for Engineering and Technology, LACCEI 2015, Santo Domingo, República Dominicana, julio 29-31, 2015, M. Larrondo Petrie y H. Álvarez, Eds., Boca Ratón: LACCEI, 2105, 141, [En línea]. Disponible: http://www.laccei.org/LACCEI2015-SantoDomingo

[8] J. Puig, M. Gijón, X. Martin y L. Rubio, "Aprendizaje-servicio y educación para la ciudadanía," Revista de Educación, número extraordinario 2011, pp. 45-67, 2011.

[9] República del Ecuador. Asamblea Nacional., Ley Orgánica de Educación Superior, Quito: Asamblea Nacional, 2010.

[10] E. Larrea de Granados, La Educación Superior desde la Complejidad Sistémica. Algunas Consideraciones para Orientar el Proceso de Construcción del Nuevo Modelo de Formación Universitaria, Quito: CES, 2014.

[11] Naciones Unidas. Asamblea General., Transformar Nuestro Mundo: la Agenda 2030 para el Desarrollo Sostenible, Nueva York: ONU., 2015.

[12] Naciones Unidas. Organización de las Naciones Unidas para la Educación, la Ciencia y la Cultura., Convención Sobre la Protección y Promoción de la Diversidad de las Expresiones Culturales, París: UNESCO, 2005.

[13] República del Ecuador. Secretaría Nacional de Planificación y Desarrollo., Plan Nacional del Buen Vivir 2013-2017, Quito: SENPLADES, 2013.

[14] Naciones Unidas. Organización de las Naciones Unidas para la Educación, la Ciencia y la Cultura., World Heritage + Sustainable Tourism Action Plan 2013-2015, París: UNESCO, 2013.

[15] Naciones Unidas. Organización de las Naciones Unidas para la Educación, la Ciencia y la Cultura., Plan de Acción para el Patrimonio Mundial en América del Sur 2015-2020, Cuzco: UNESCO, 2015.

[16] T. Erice, "Patrimonio inmaterial y gastronomía: historia de una relación," Primer Simposio Gastronómico del Guayas 'Memoria e Identidad Cultural', enero 27, 2016, Guayaquil, Ecuador, inédito.

[17] T. Erice, "Ethnohistory at the Archivo Histórico del Guayas (AHG): Bridging the Gap Between Prehispanic Archaeology and Colonial History," Archaeol. Rev. Camb., vol. 29, no. 2, pp. 88-102, noviembre 2014.

[18] K. Kottak, Antropología Cultural, México D.F.: Mc Graw-Hill, 2011, p. 19.

[19] M. Augé, El Sentido de los Otros. Actualidad de la Antropología, Barcelona: Paidós, 1996. 
[20] J. Pujadas i Muñoz, D. Comas d'Argemir y J. Roca i Girona, Etnografia, Barcelona: UOC, 2010.

[21] C. Banderas, C. López, A. Sáenz de Viteri, M. Icaza y D. Torres, Guayas: Entre sabores y saberes Patrimoniales. Naranjal y Balao, Guayaquil: ESPOL, 2016. [En línea] Disponible: http://www.espol.edu.ec/sites/default/files/espol/DOSSIER.pdf

[22] J. Prat Ferrer, "Sobre el concepto de folklore," Oppidum, vol. 2, pp. 229248,2006

[23] Naciones Unidas. Organización de las Naciones Unidas para la Educación, la Ciencia y la Cultura, Convención para la Salvaguarda del Patrimonio Cultural Inmaterial, París: UNESCO, 2003.

[24] República del Ecuador. Secretaría Nacional de Ciencia y Tecnología., Plan Nacional de Ciencia, Tecnología, Innovación y Saberes Ancestrales, Quito: SENACYT, 2010.

$14^{\text {th }}$ LACCEI International Multi-Conference for Engineering, Education, and Technology: "Engineering Innovations for Global Sustainability", 20-22 July 2016, San José, Costa Rica. 